\title{
A COMBINATORIAL APPROACH TO THE OPPOSITE BI-FREE PARTIAL S-TRANSFORM
}

\author{
PAUL SKOUFRANIS
}

Abstract. In this paper, we present a combinatorial approach to the opposite 2-variable bi-free partial $S$-transforms where the opposite multiplication is used on the right. In addition, extensions of this partial $S$-transforms to the conditional bi-free and operator-valued bi-free settings are discussed.

Mathematics subject classification (2010): 46L54, 46L53.

Keywords and phrases: Bi-free probability, partial bi-free $S$-transform, bi-free convolutions.

\section{REFERENCES}

[1] I. Charlesworth, B. Nelson, P. Skoufranis, Combinatorics of Bi-Free Probability with Amalgamation, Comm. Math. Phys. 338, 2 (2015), 801-847.

[2] I. Charlesworth, B. Nelson, P. Skoufranis, On two-faced families of non-commutative random variables, Canad. J. Math. 67, 6 (2015), 1290-1325.

[3] K. Dy Kema, On the S-transform over a Banach algebra, J. Funct. Anal. 231, 1 (2006), 90-110.

[4] Y. Gu, H.-W. HuAng, J. Mingo, An analogue of the Lévy-Hinčin formula for bi-free infinitely divisible distributions, to appear in Indiana Univ. Math. J., (2015), 26 pages.

[5] Y. Gu, P. Skoufranis, Conditional Bi-Free Independence for Pairs of Algebras, arXiv:1609.07475, (2016), 44.

[6] Y. Gu, P. Skoufranis, Conditional Bi-Free Independence with Amalgamation, arXiv:1609.07820, (2016), 36.

[7] Y. GU, P. Skoufranis, Bi-Boolean independence for pairs of algebras, arXiv:1703.03072, (2017), 42.

[8] U. HAagerup, On Voiculescu's $R$ - and $S$-transforms for free non-commuting variables, "Free Probability Theory", D. V. Voiculescu, editor, Fields Institute Communications 12 (1997), 127-148.

[9] H.-W. Huang, J.-C. Wang, Analytic Aspects of Bi-Free Partial R-Transforms, J. Funct. Anal. 271, 4 (2016), 922-957.

[10] H.-W. Huang, J.-C. WAng, Harmonic Analysis for the Bi-Free Partial S-Transform, preprint, (2017), 28 pages.

[11] A. NiCA, R. SPEICHer, A "Fourier Transform" for Multiplicative Functions on Non-Crossing Partitions, J. Algebraic Combin. 6 (1997), 141-160.

[12] M. Popa, J. C. WANG, On multiplicative conditionally free convolution, Trans. Amer. Math. Soc. 363, 12 (2011), 6309-6335.

[13] P. Skoufranis, A Combinatorial Approach to Voiculescu's Bi-Free Partial Transforms, Pacific J. Math. 2, 283 (2016), 419-447.

[14] P. Skoufranis, Independences and Partial R-Transforms in Bi-Free Probability, Ann. Inst. Henri Poincaré Probab. Stat. 52, 3 (2016), 1437-1473.

[15] P. Skoufranis, On Operator-Valued Bi-Free Distributions, Adv. Math. 303 (2016), 638-715.

[16] R. SPEICHER, Multiplicative functions on the lattice of non-crossing partitions and free convolution, Math. Ann. 298, 1 (1994), 611-628.

[17] D. Voiculescu, Addition of certain non-commuting random variables, J. Funct. Anal. 66, 3 (1986), 323-346. 
[18] D. VOICULESCU, Multiplication of certain non-commuting random variables, J. Operator Theory 18 (1987), 223-235.

[19] D. Voiculescu, Free probability for pairs of faces I, Comm. Math. Phys. 332, (2014), 955-980.

[20] D. V. Voiculescu, Free Probability for Pairs of Faces II: 2-Variable Bi-Free Partial R-Transform and Systems with Rank $\leqslant 1$ Commutation, Ann. Inst. Henri Poincaré Probab. Stat. 52, 1 (2016), 1-15.

[21] D. V. VOICULESCU, Free probability for pairs of faces III: 2-variable bi-Free partial $S$-transform and T-transforms, J. Funct. Anal. 270, 10 (2016), 3623-3638. 